\title{
The hollow fibre model - facilitating anti-cancer pre-clinical pharmacodynamics and improving animal welfare
}

\author{
MARIE SUGGITT, PATRICIA A. COOPER, STEVEN D. SHNYDER and MICHAEL C. BIBBY \\ Institute of Cancer Therapeutics, University of Bradford, Bradford, BD7 1DP, UK
}

Received April 7, 2006; Accepted May 29, 2006

\begin{abstract}
We describe a modified hollow fibre assay (HFA) for investigating the potential of novel molecules as pharmaceutical agents. In particular the assay provides drug/target interaction data that can facilitate the selection of lead compounds for further evaluation in more sophisticated solid tumour models, whilst successfully implementing the 3Rs - the 'replacement' 'refinement' and 'reduction' of animals. This more ethical and rapid approach to early drug development does not compromise on the validity, sensitivity, predictivity or efficacy of preclinical evaluation. We present novel data using the standard cross-linker mitomycin C (MMC) as a positive control, and two investigational DNA interactive molecules (C1311/ SJG-136). Tumour cells were seeded in fibres and implanted into mice. Following treatment with an intraperitoneal injection, fibres were excised and cells retrieved for pharmacodynamic analysis using the comet assay/fluorescence microscopy. Microscopy results revealed nuclear uptake and localisation within cytoplasmic organelles of HT29 colorectal adenocarcinoma cells following treatment with C1311 (150 mg/kg). Following treatment with SJG-136 $(0.3 \mathrm{mg} / \mathrm{kg})$ a $27.3 \%(\mathrm{p}<0.001)$ DNA cross-linking (s.c.) effect was observed in the HL60 acute promyelocytic leukaemia cell line. DNA cross-linking effects of $55 \%$ (i.p) and $50 \%$ (s.c.) $(\mathrm{p}<0.005)$ were observed in the A549 lung carcinoma cell line following administration of MMC $(6 \mathrm{mg} / \mathrm{kg})$. These data are consistent with previous activity defined using solid tumour models, and support the use of the HFA for in vivo pharmacodynamic investigation whilst significantly reducing animal numbers and the influence of tumour growth on the welfare of mice.
\end{abstract}

\section{Introduction}

The pre-clinical testing of anti-cancer agents prior to clinical registration is steeped in a history of using considerable numbers of animals. The philosophy of the 'replacement, refinement and reduction' (3Rs) of animals in research first documented by Russell and Burch in 1959 (1) has influenced

Correspondence to: Dr Marie Suggitt, Institute of Cancer Therapeutics, University of Bradford, Bradford, BD7 1DP, UK

E-mail: marie.suggitt@yahoo.co.uk

Key words: hollow fibre assay, pharmacodynamics, 3Rs new legislation aimed at controlling the use of animals. The 3Rs have since been formally incorporated in the Animal (Scientific) Procedures Act, 1986 (UK) and the Animal Welfare Act, 1998 (USA). In September 2004 a national centre for the replacement, refinement and reduction of animals in research 'NC3Rs' was established in the UK which aims to increase the development and implementation of the $3 \mathrm{Rs}$ in biomedical, biological and veterinary research in academia and industry.

Both the NC3Rs and the Interagency Coordinating Committee on the Validation of Alternative Methods (ICCVAM) (USA) (2) aim to fulfil the need for considering the humane treatment of animals whilst maintaining scientific credibility. Despite the awareness of federal regulatory and research agencies [e.g. National Cancer Institute (NCI) and Food and Drug Administration] very little literature documenting precise methods in which to implement the $3 \mathrm{Rs}$ philosophy is available. We believe that adherence to the $3 \mathrm{Rs}$ philosophy should be a very important factor considered when planning animal experimentation, and we present the hollow fibre assay (HFA) as a means of implementing the $3 \mathrm{Rs}$ in early pre-clinical drug development.

The HFA was developed by the NCI as a low cost, high-throughput, preliminary in vivo screening assay for the evaluation of anti-cancer agents (3). It has since been adapted for the evaluation of antiviral agents (4), and there is considerable scope for use in other medical fields.

Since 1995 the NCI protocol has involved the short-term in vitro culture (24-48 h) of cells inside biocompatible hollow fibres, followed by implantation at both subcutaneous (s.c) and intraperitoneal (i.p) sites of the nude mouse (3 fibres/site). Mice are treated with test compound at two doses for up to 4 days, fibres excised and cells assessed for 'viability' using a modified MTT (3-[4,5-dimethylthiazol-2-yl]-2,5-diphenyltetrazolium bromide) assay $(3,5)$. Optimal or near-optimal treatment regimens are indicated for further testing using xenograft models (3).

Due to the practicality of being able to implant up to six different cell lines in each host (3/site) the NCI HFA requires only 24 mice for the testing of one agent against a panel of 12 cell lines. In contrast the NCI initially use three xenograft models to test one agent with each xenograft model requiring $\sim 50$ mice.

In line with the transition from the development of classical cytotoxic drugs to target-orientated drug discovery (6), more recently the HFA has been used to investigate the pharmacodynamics (drug-target interaction) of anti-cancer agents in vivo. Researchers are taking advantage of the practical feasibility 
of pure tumour cell retrieval from fibres and have used a variety of techniques to evaluate pharmacodynamic (PD) activity (7-11). In contrast, solid tumour models are not so amenable to pure cell retrieval due to the presence of host cells, and a laborious protocol is required before performing PD analysis. Recently the HFA has also been developed for studying agents that target the tumour neovasculature (12). The HFA was not developed to recapitulate the complexities of human cancer or to reproduce the complex interactions of the host cell/tumour micro-environment. It is proposed as an ideal in vivo method in which to demonstrate proof of principle activity or indeed to eliminate agents not shown to interact with their respective targets in vivo at an early pre-clinical stage. Agents identified as active in the HFA may progress and undergo more comprehensive pharmacokinetic/PD studies using xenograft models, but this initial evaluation using the HFA will still lead to considerable savings in animal numbers.

We describe novel pharmacodynamic end-points of the in vivo HFA, namely DNA cross-linking and drug uptake/ cellular localisation evaluated using the COMET assay and fluorescence microscopy. Both standard and investigational agents, MMC, SJG-136, and C1311 will be used.

Mitomycin C is a DNA cross-linking agent that has been used in the clinic for over 20 years. It is used as a primary chemotherapy agent in the treatment of anal, lung and superficial bladder cancers and also as a secondary agent in breast, colon, gastric and pancreatic cancers.

SJG-136 is a recently synthesised pyrrolo[2,1-c][1,4]benzodiazepine (PBD) dimer currently undergoing phase I clinical trial in both the UK (CRUK) and USA (NCI). The pyrrolo[2,1c] $[1,4]$ benzodiazepines are a family of tricyclic antitumour antibiotics which interact sequence selectively with purineguanine-purine motifs in the minor groove of DNA, reacting covalently with the exocyclic $\mathrm{C}_{2}-\mathrm{NH}_{2}$ of the central guanine via their electrophilic imine moiety (13).

C1311 is a member of a group of imidazoacridinones that have been developed in an attempt to generate antineoplastic agents combining the common characteristics of acridines and anthracyclines essential for anti-cancer activity (e.g. planar structure and polycyclic nucleus) with high affinity to target DNA and resistance to production of free radicals by enzymatic reduction (14). Ultimately we aim to demonstrate the use of the HFA for PD investigation in vivo whilst successfully implementing the philosophy of the 3Rs.

\section{Materials and methods}

Cell culture. All cell culture procedures were carried out in sterile microflow class II biological safety cabinets (MDH). A549 lung carcinoma (15), HL60 acute promyelocytic leukaemia (16), and HT29 colorectal adenocarcinoma (17) cell lines previously confirmed as mycoplasma-free, were cultured using RPMI-1640 medium (Sigma-Aldrich, St. Louis, MO) containing 10\% fetal calf serum (FCS) (SigmaAldrich-Aldrich), $1 \%$ penicillin/streptomycin $(50 \mu \mathrm{g} / \mathrm{ml})$ (Sigma-Aldrich), 1\% L-glutamine (2 mM) (Sigma-Aldrich), and $1 \%$ sodium pyruvate $(1 \mathrm{mM})$ (Sigma-Aldrich). Cells were grown in $75 \mathrm{~cm}^{3}$ flasks (Costar ${ }^{\circledR}$ High Wycombe, UK) in a humidified incubator at $37^{\circ} \mathrm{C}$ with $5 \% \quad \mathrm{CO}_{2}$ (Heraeus Instruments, South Plainfield, NY).
Hollow fibre preparation. All hollow fibre procedures were based on those of Hollingshead et al (3). Polyvinylidine fluoride (PVDF) hollow fibres (Type f) (Spectrum ${ }^{\circledR}$ Laboratories Inc., Houston, TX) with a $500 \mathrm{kDa}$ mw cut-off, and $1 \mathrm{~mm}$ inner diameter were used. Tumour cells were cultured as described above, a subconfluent flask of cells attained and seeded at the desired density into hollow fibres. Fibres were placed in a $75 \mathrm{~cm}^{3}$ (Costar) flask of RPMI-1640 medium (20\% FCS) and incubated at $37^{\circ} \mathrm{C}\left(5 \% \mathrm{CO}_{2}\right)$ overnight.

In vivo implantation of hollow fibres. Pure strain female NMRI mice (B\&K Universal Ltd., Hull, UK) or NCR/Nu (NCI, Frederick, USA) mice aged 6-8 weeks were used. All mice had access to food (CRM diet, SDS, Witham, Essex, UK) and water ad libitum. All animals were kept in cages in an air-conditioned room with alternating cycles of light and dark. All animal studies were carried out under a Home Office licence. UKCCCR guidelines for the welfare of animals in experimental neoplasia were adhered to throughout the study (18). Mice were anaesthetised by inhalation of isoflurane (Rhodia Organique Fine Ltd.). Three fibres were implanted at both subcutaneous (s.c.) and intraperitoneal (i.p.) sites. Three mice were used per treatment group. Incisions (i.p. and s.c.) were closed with histoacryl tissue glue (B/braun Surgical, Barcelona, Spain), with an additional skin staple.

Tumour cell retrieval from hollow fibres. Twenty-four hours following treatment mice were sacrificed by cervical dislocation, fibres excised, wiped to remove excess host tissue and transferred to pre-warmed RPMI (10\% FCS) medium for $30 \mathrm{~min}$. Fibres were transferred to $1 \mathrm{ml}$ of pre-warmed accutase solution (Innovative Cell Technologies; in Dulbecco's phosphate buffered saline $0.5 \mathrm{mmol} / \mathrm{l}$ EDTA) in 6-well plates. Fibre ends were cut and discarded. Fibres were flushed through once with the $1 \mathrm{ml}$ accutase solution. Plates were then incubated at $37^{\circ} \mathrm{C}\left(5 \% \mathrm{CO}_{2}\right)$ for $30-45 \mathrm{~min}$ on an orbital shaker at $100 \mathrm{~g}$ (Lab-Line Instruments, Melrose Park, IL) to detach cells from fibre walls and attain a single cell suspension ready for the comet assay or microscopy analysis.

Assessment of tumour cell growth within hollow fibres. Prior to performing pharmacodynamic investigations tumour cell growth within hollow fibres was characterised so that linear growth could be defined. On each day of growth assessment mice were sacrificed by cervical dislocation, fibres excised, and a modified version of the MTT (3-[4,5-dimethylthiazol2-yl]-2,5-diphenyl-tetrazolium bromide (tetrazolium) dye conversion assay performed (3). Absorbance was measured at $540 \mathrm{~nm}$ using a spectrophotometer (Multiscan Plus, Labsystems, Life Sciences International Ltd., Basingstoke, UK., Genesis Labsystems software version 3.04). The percentage of net cell growth was calculated as follows:

Mean absorbance on retrieval day - Mean absorbance on implantation day x 100 Mean absorbance on implantation day

Chemotherapy. Mitomycin C (M.W. 334.3) (Sigma-Aldrich), and C1311 (M.W 350) (Xanthus Life Sciences) were dissolved in physiological saline and administered at previously defined 
maximum tolerated doses (MTDs)/therapeutic doses of $6 \mathrm{mg} / \mathrm{kg}$ (19) and $150 \mathrm{mg} / \mathrm{kg}(20,21)$ respectively.

SJG-136 (M.W 557.1), a gift from Professor D. Thurston (School of Pharmacy, University of London, UK) was dissolved in 5\% dimethyl-acctamide/saline and administered at a known therapeutic dose of $0.3 \mathrm{mg} / \mathrm{kg}$ (22). All compounds were given $0.1 \mathrm{ml}$ per $10 \mathrm{~g}$ bodyweight by a single intraperitoneal injection.

Alkaline single cell gel electrophoresis assay (Comet Assay). Comet assay procedures were based on those of ref. 23. At specific time-points following treatment with MMC/SJG-136 mice were sacrificed, fibres excised and tumour cells prepared as described above for analysis. Mice were sacrificed $24 \mathrm{~h}$ and $2 \mathrm{~h}$ following treatment with SJG-136 and MMC respectively as MMC-induced DNA cross-links are known to be repaired relatively rapidly compared to SJG-136-induced DNA crosslinks which are thought to be irreversible. Each cell suspension was pelleted and incubated with $\mathrm{H}_{2} \mathrm{O}_{2}(100 \mu \mathrm{M})\left(4^{\circ} \mathrm{C}\right),(20 \mathrm{~min})$ (24) to introduce single strand breaks. Cell suspensions were pelleted and resuspended in $150 \mu \mathrm{l}$ PBS. Many electrophoretic methods for the separation of DNA containing an interstrand crosslink are based on the principle that such a lesion can prevent the complete denaturation of the two DNA strands. Therefore as the number of crosslinks increases the amount of DNA able to migrate is reduced. Thus crosslinks are quantified as the decrease in comet tail moment compared with untreated controls.

Frosted edge slides were placed into normal melting point (NMP) agarose $(1 \%)\left(45^{\circ} \mathrm{C}\right)$, removed, the underside wiped and allowed to dry overnight. All procedures from here were carried out in a dark room under red light. Low melting point agarose $(150 \mu \mathrm{l})(\mathrm{LMA})(1 \%)\left(45^{\circ} \mathrm{C}\right)$ was added to each cell suspension. Each cell suspension $(150 \mu \mathrm{l})$ was added to two agarose coated slides, a coverslip placed on each, and slides placed on ice for $\sim 5 \mathrm{~min}$. The coverslip was removed and $150 \mu 1$ of LMA $(0.5 \%)$ was added as a third layer for protection of cells. The coverslip was replaced and the slide placed again on ice for $5 \mathrm{~min}$.

Coverslips were removed and all slides gently placed in a slide rack in cold-lysis solution $(2.5 \mathrm{M} \mathrm{NaCl}, 100 \mathrm{mM}$ ethylenediaminetetraacetic acid (EDTA), and $10 \mathrm{mM}$ Trizma ${ }^{\circledR}$ Base $\mathrm{pH} 10.0)$ for a minimum of $1 \mathrm{~h}\left(4^{\circ} \mathrm{C}\right)$. Dimethyl sulfoxide (10\%) (DMSO) and $2 \mathrm{ml}$ of Triton X-100 was added to lysing solution. Slides were placed in a horizontal electrophoresis gel tank and covered with electrophoresis buffer $(1 \mathrm{mM}$ EDTA and $300 \mathrm{mM} \mathrm{NaOH}, \mathrm{pH}>13.0$ ), and incubated for $30 \mathrm{~min}$ to unwind DNA. DNA was then electrophoresed at $25 \mathrm{~V}$ for $30 \mathrm{~min}$ (CONSORT $600 \mathrm{~V}-1000 \mathrm{~mA}$ E861).

Slides were removed and washed with neutralizing buffer (TrizmaBase pH 7.5) for 5 min. Slides were covered in PBS for $5 \mathrm{~min}$. Fresh PBS was added for an additional $5 \mathrm{~min}$. Slides were air-dried overnight, and placed in distilled water for $30 \mathrm{~min}$.

Slides were stained with SYBR ${ }^{\circledR}$ gold (x10,000 in distilled water) (Molecular Probes, Carlsbad, CA) (500 $\mu 1 /$ slide) and incubated for $30 \mathrm{~min}$. Slides were placed under a fluorescence microscope (LEICA DMLB). Using Comet III - Perceptive Instruments (Suffolk, UK) software, connected to the fluorescence microscope and camera, cells were counted and analysed. Cells (25) were counted randomly from one slide and another 25 from the replicate slide.

Tail moment (measurement of tail length $\mathrm{x}$ measurement of DNA in the tail) was used as a primary measure of DNA damage. Percentage DNA cross-linking was determined using the following calculation:

100 - (mean treated comet value/mean control value $\mathrm{x} 100$ )

Fluorescence microscopy. At $4 \mathrm{~h}$, mice were sacrificed by cervical dislocation, fibres excised and prepared as described above. Single cell suspension was pelleted, resuspended in RPMI medium, and pipetted onto to glass slides and a coverslip placed on top. Images of C1311 cellular uptake and localisation were captured using a confocal microscope (Biorad Microradiance Confocal Imaging System) attached to a Nikon CM-800 microscope using Laser Sharp 2000 software (excitation/emission maxima 340/520 nm).

Tissue processing and staining of fibre sections. Fibres were retrieved from mice and placed in $10 \%$ buffered-formalin for $\sim 4 \mathrm{~h}$, and transferred to $70 \%$ ethanol until required for processing. Fibres were embedded in paraffin in a Leica TP 1020 automatic tissue processor (Leica, Milton Keynes, UK). Fibres were removed from the tissue processor, aligned in small plastic cubes and filled with hot paraffin wax on an embedding centre (Raymond Lamb, East Sussex, UK). Cubes were placed on a cold plate and left over night to solidify before being sectioned. Sections $(10 \mu \mathrm{m})$ were cut using a rotary microtome, mounted on glycerine albumen (Merck) coated slides, and allowed to dry overnight. Slides were submerged sequentially in haematoxylin (10 min), (washed, tap water), acid alcohol ( $0.5 \%$ hydrochloric acid in $70 \%$ ethanol) (5 sec) (washed, tap water), Scott's tap water (2 min), $1 \%$ aqueous eosin ( $1 \mathrm{~min}$ ) (wash), $100 \%$ ethanol (1 min), ethanol (3 $\mathrm{min}), 50 \%$ xylene/ethanol (3 $\mathrm{min}), 100 \%$ xylene ( $3 \mathrm{~min}$ ) and $100 \%$ xylene (5 min). Several drops of DPX were added to a coverslip, placed on each slide, and allowed to air dry. Slides were then ready to be observed under the light microscope.

\section{Results}

Characterisation of tumour cell growth within hollow fibres in immuno-competent and immuno-deficient hosts. We present data demonstrating that human tumour cells grow equivalently in both immuno-competent NMRI and immunodeficient athymic nude hosts (Fig. 1). HT29 colorectal adenocarcinoma cells were seeded in hollow fibres and implanted in both hosts over seven days. At each time-point mice were sacrificed, fibres excised and the MTT assay performed to assess cell viability. Raw absorbance values derived from fibres (3/site) retrieved from both hosts (s.c/i.p) were compared at each time-point. No significant difference $(\mathrm{p}>0.05)$ in raw absorbance values was observed between NMRI immunocompetent and nude immuno-deficient mice on days 3, 5 and 7 (i.p/s.c). Fig. 1 presents mean percentage net cell growth data derived from raw absorbance values. Fig. 1 insert shows the morphology of HT29 cells growing along the internal surface of the fibre in vivo. 


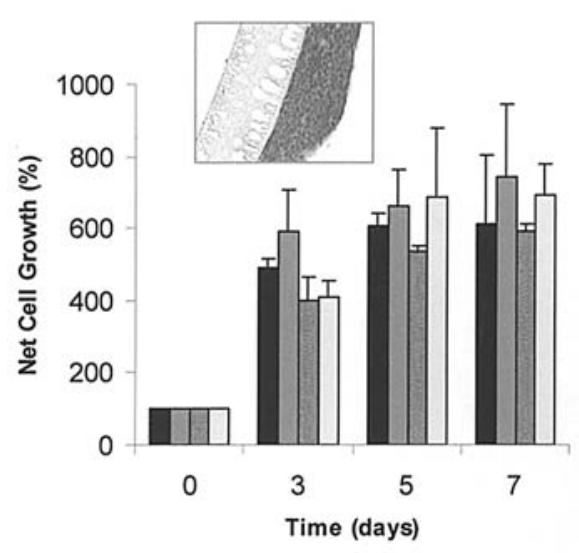

aMRI ip $\square$ Nude ip $\square$ NMRI sc $\square$ Nude sc

Figure 1. Net cell growth (\%) of HT29 colon carcinoma cells grown in hollow fibers implanted in nude and NMRI mouse strains. Tumor cells were seeded at $1 \times 10^{6}$ cells $/ \mathrm{ml}$ in hollow fibers and incubated overnight $\left(37^{\circ} \mathrm{C}\right)$ $\left(5 \%, \mathrm{CO}_{2}\right)$ in RPMI media $(20 \%$ FCS $)$. Fibers were implanted into mice at s.c. and i.p. sites. At each time-point mice were sacrificed, fibers excised and the MTT assay performed to determine cell viability. Results are expressed as mean \% net cell growth. Insert, HT29 colon carcinoma cells growing along the internal surface of the fibre at the s.c. site in NMRI mouse.

DNA cross-linking assay. The Comet assay was used to assess the pharmacodynamic activity of the standard cross-linking agent MMC following treatment of NMRI fibre-bearing mice at the MTD. Fig. 2 presents frequency histograms of DNA comet tail moments and comet images following analysis of A549 non-small cell lung carcinoma cells retrieved from fibres $2 \mathrm{~h}$ post treatment. Mean tail moments calculated from
MMC-treated mice were 31.96 \pm 25.56 and 31.5 \pm 23.12 at i.p. and s.c sites respectively. Mean values of cells retrieved for vehicle-treated mice were $71.49 \pm 32.1$ and $62.3 \pm 30.28$ at i.p. and s.c. sites respectively. Differences in tail moment distribution between control and treatment groups at both i.p and s.c sites are highly significant $(p<0.005$, Mann Whitney test). Percentage MMC-DNA cross-linking values were calculated at $55.29 \%$ and $49.44 \%$ for i.p. and s.c. sites respectively.

The pharmacodynamic activity of the investigational agent SJG-136 was also evaluated using the comet assay. Fig. 3 presents DNA frequency histograms of comet tail moments and comet images of HL60 leukemia cells retrieved from hollow fibres (s.c) $24 \mathrm{~h}$ following treatment with the MTD. HL60 cells retrieved from SJG-136- and vehicle-treated mice were shown to have mean tail moment values of $16 \pm 9$ and $22 \pm 12$ respectively $(\mathrm{p}<0.001$ Mann Whitney test). A $27.3 \%$ DNA cross-linking effect of SJG-136 was calculated.

Fluoresence microscopy. NMRI fibre-bearing mice were treated with the imidazoacridinone C1311 (150 mg/kg). At $4 \mathrm{~h}$ after treatment, fibres were excised and HT29 cells retrieved for fluorescence microscopy analysis. Nuclear localisation was observed along with punctate concentrations evident in cytoplasmic organelles (Fig. 4).

\section{Discussion}

This study has successfully characterised the HFA for investigating novel pharmacodynamic end-points in vivo, namely DNA cross-linking and drug uptake/cellular localisation, whilst implementing the philosophy of the 3Rs. a)
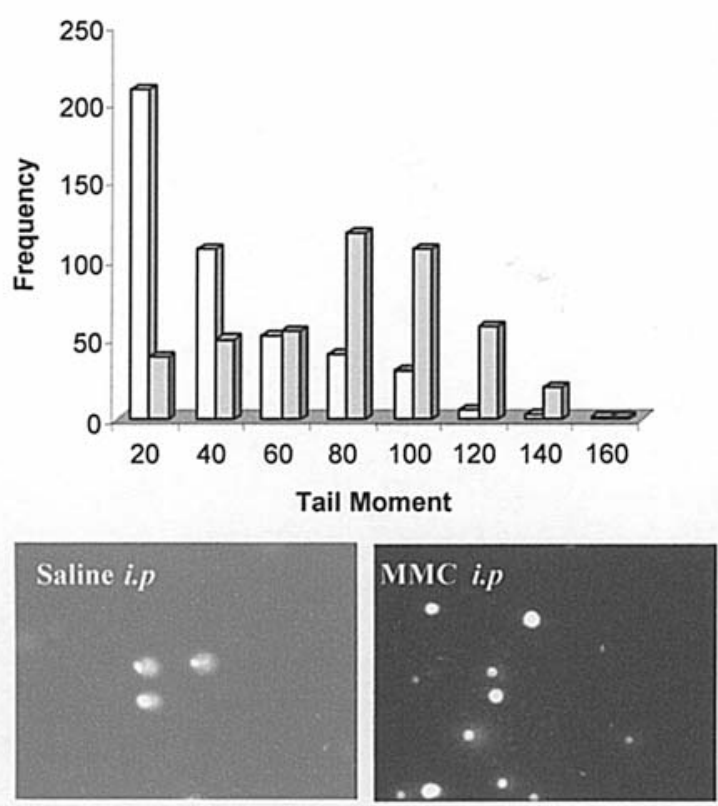

b)
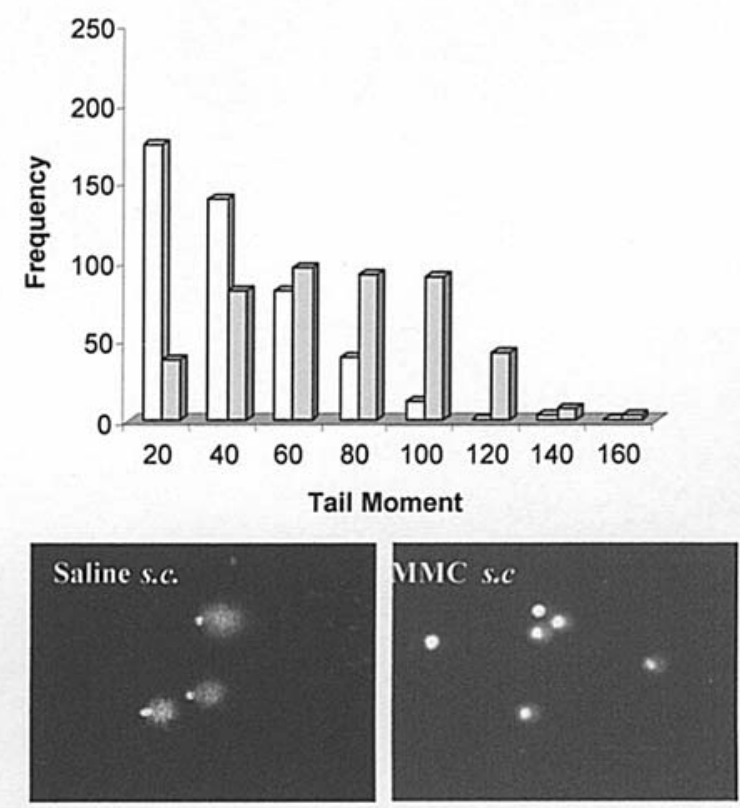

Figure 2. Frequency histograms of DNA comet tail moments. A549 non-small cell lung carcinoma cells were seeded in hollow fibers (1x10 6 cells/ml), cultured in vitro overnight, and implanted into NMRI mice at both a, i.p; and b, s.c. sites. On day 3 mice were treated with either MMC (white bars) or physiological saline (grey bars) at $6 \mathrm{mg} / \mathrm{kg}$ by a single i.p. injection. At $2 \mathrm{~h}$ mice were sacrificed, cells retrieved from fibers and the comet assay performed. Each set of bars represents the analysis of 450 cells ( 3 mice x 3 fibers/site x 50 comets). 


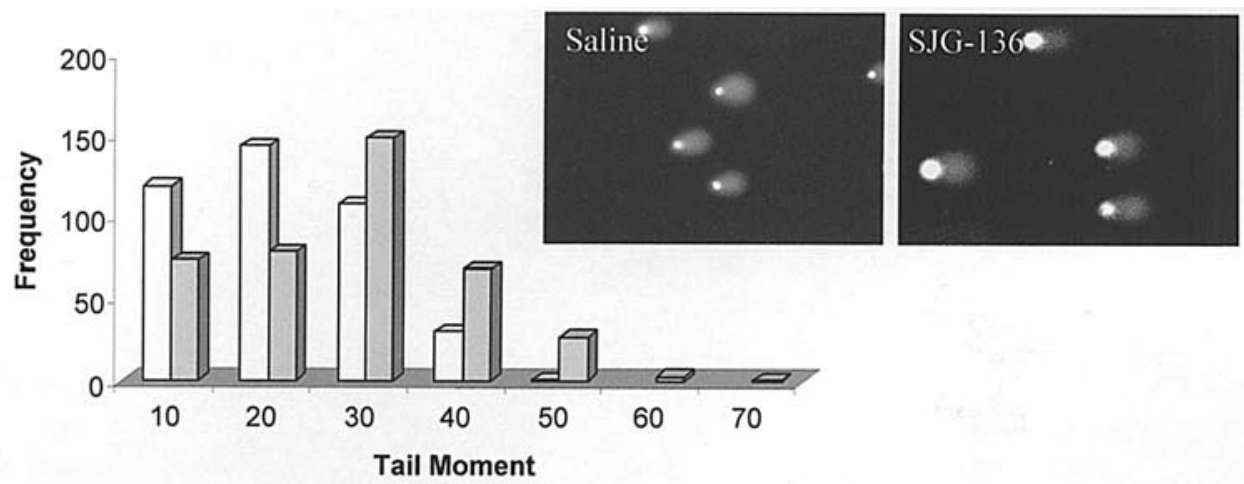

Figure 3. DNA frequency histogram of comet tail moments. HL60 leukemia cells were seeded into hollow fibers (1x10 ${ }^{6}$ cells/ml) and implanted into NMRI mice at the s.c. site. On day 4 mice were treated with either SJG-136 (white bars) or 5\% dimethyl-acetamide/saline vehicle (grey bars) at an MTD of 0.3 $\mathrm{mg} / \mathrm{kg}$ by a single i.p. injection. At $24 \mathrm{~h}$ mice were sacrificed, cells retrieved and the comet assay performed. Each set of bars represents the analysis of 450 cells ( 3 mice $\mathrm{x} 3$ fibers site $\mathrm{x} 50$ comets).
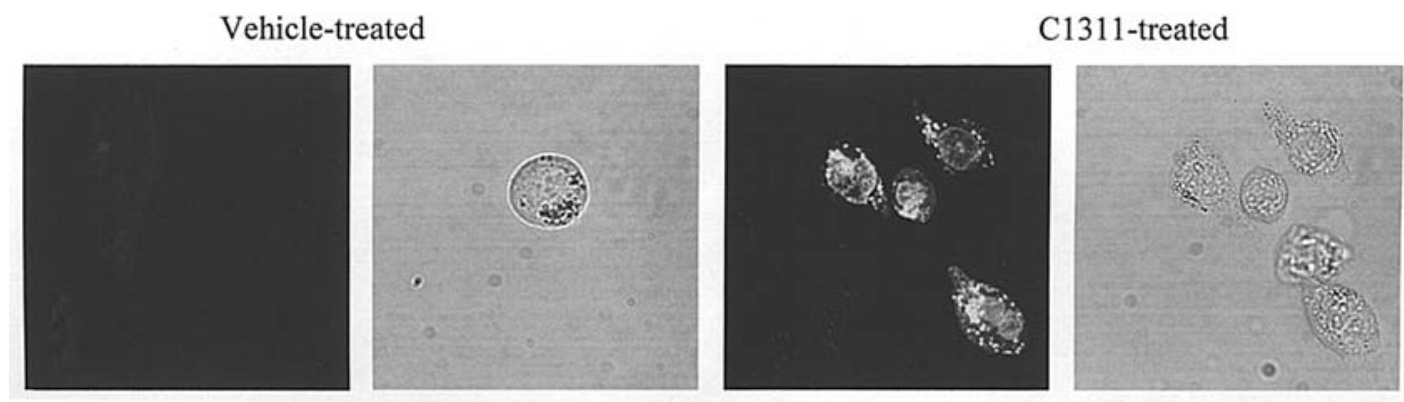

Figure 4. Fluorescence microscopy images: HT29 cells were seeded in hollow fibers (2x106 cells/well), incubated overnight and implanted into the i.p. site of NMRI mice. On day 3 mice were treated with C1311 or physiological saline (150 mg/kg) (i.p.). Mice were sacrifled at 4 h, fibers excised and cells observed using fluorescence microscopy. Fluorescence images of HT-29 cells retrieved from vehicle-treated mice are also presented so that the effects of autofluorescence can be eliminated. Both fluorescence and transmission images are presented. Images are representative of whole tumor cell populations.

MMC has previously been found to be active using the HFA as defined by the routine MTT assay (9). Here the HFA has been used to confirm, for the first time the proposed PD activity of the standard agent MMC in vivo. This in vivo HFA activity supports previous activity defined using a breast xenograft model (25).

The PBD dimer SJG-136 has recently generated a score of 54/96 using the NCI routine HFA (22) and has demonstrated significant anti-tumour activity against several human tumour xenograft models including a HL-60 tumour model (26). Here the HFA has confirmed the proposed cross-linking activity of SJG-136 in vivo.

The cellular uptake and localisation in vitro $(20,21,27)$ and activity in both murine and HT29 human tumour xenograft models (20) of the imidazoacridinone C1311 has previously been demonstrated. Rapid nuclear and lysosomal uptake was observed (27). Lysosomal swelling and breakdown have been shown to precede apoptosis, and it is thought that these lyso-osmotropic effects of C1311 may be a novel feature of this anti-cancer agent (27). C1311 has also previously been evaluated using the routine in vivo HFA, revealing a 68 and $57 \%$ growth inhibition in HT-29 cells respectively at the i.p. and s.c. site respectively (Personal communication: Jesse Paterson, Xanthus Life Sciences). Here we have used the HFA to demonstrate cellular localisation of C1311 for the first time in vivo, supporting previous in vitro and in vivo anti-tumour xenograft studies.
The results of our DNA crosslinking and drug uptake/ cellular localisation pharmacodynamic studies collectively demonstrate the comparable activity generated in both HF (using 3 mice/group) and previous xenograft models.

Our laboratory has also demonstrated the selective PD activity of 'Phortress', a DNA reactive 2-(4-amino-3-methylphenyl)-5-fluorobenzothiazole prodrug currently in phase I trials, using both HFA and xenograft models (8). PD activity has also been demonstrated using Combretastatin A-1 phosphate (CA1P) in the HFA in vivo (7), supporting previous anti-tumour activity demonstrated using xenograft models (28). Known clinically active antiviral agents have been shown to suppress infection in both HFA and xenograft models (4), again demonstrating the comparable data defined by both xenograft and the HF models.

With respect to the development of cytotoxic agents, the traditional s.c. xenograft model appears to have value as a predictive in vivo preclinical model (29), yet it remains to be seen if the xenograft will retain its predictive capacity in this modern era of rationally designed small molecules. Whether alternative models (e.g. genetically engineered/transgenic/ knockout) may be more predictive of clinical response or provide a more clinically relevant model is yet to be elucidated (30).

The HFA has consistently been shown to have good predictivity of xenograft activity $(6,31,32)$. All agents that have progressed to the clinic for routine use that have been 
tested in both xenograft and HF models have been found to be active in both models. Additionally, agents under clinical investigation identified as active in the NCI routine HFA, have also been tested extensively in xenograft models (e.g. Phortress, C1311, SJG-136, and the proteosome inhibitor PS341).

In recognition of the heterogeneity of clinical disease, it is believed that for a pre-clinical model to predict clinical activity it must possess the relevant molecular drug-target. Agents identified as active in molecularly characterised preclinical models must be rationally administered to patient populations possessing the relevant biomarker. Considering that many non-toxic therapies may be cytostatic it has been suggested that early phase clinical trials may need to incorporate measures of anti-tumour efficacy other than tumour size, for instance measurement of target inhibition (33). Parulekar and Eisenhauer's review of 60 recent phase I trials of noncytotoxic agents clearly indicated that these data were lacking.

If drug-target interaction is to be considered as a biomarker in the clinic then it seems plausible to use a practically amenable in vivo model, namely the HFA (appropriately characterised for the relevant PD end-point) as a predictor of clinical activity. Such application may even speed up the selection of lead compounds for clinical trial.

Such pharmacodynamic application of the HFA permits the follow-up of a compound's mechanisms in vivo following its indication in vitro by molecularly characterised in vitro screens.

With respect to the $3 \mathrm{Rs}$, implementing the principle of 'reduction' has caused concern with respect to using too few animals that could lead to inconclusive results $(34,35)$. It is emphasised that each in vivo hollow fibre study uses only a maximum of 6 mice (3/group) permitting the retrieval of 9 individual tumour cell populations ( 3 i.p./s.c. x 3 mice) per group. In comparison only 1-2 individual tumour populations are derived from one xenograft mouse model. In the case of our comet assay analysis, a treatment group of 3 mice generates 450 comet tails moments (9/site x 50 comets) used for determination of mean tail moment. These numbers are sufficient to measure statistical significance.

If we take into consideration that one xenograft model would generally use 50 mice and that the HFA has determined activity comparable with that determined in xenograft models then it would seem plausible to rely on the HFA for such pharmacodynamic investigation. Simultaneously animal numbers are reduced significantly without compromising on the scientific credibility of the assay. As well as the considerable reduction in the numbers of mice required, other 3R benefits of the HFA include a shorter assay time of less than one week. In contrast routine xenograft models may take between two weeks and four months to complete depending on the end-points selected for study. Such a 'refinement' reduces the time animals are exposed to experimental procedures, and decreases the tumour burden thus reducing the release of cachectic and other deleterious material from tumour cells into the host.

These novel pharmacodynamic investigations that have successfully provided 'proof of principle' of a compounds mechanisms of action in vivo have been facilitated by the practical amenability of cell retrieval from fibres.
The debatable value of other in vivo models in predicting clinical response, evidence of good pre-clinical predictivity of the HFA, the comparable applicability of the HFA with the xenograft model, all lend support for the more extensive use of the HFA.

In this era of rational drug design the hollow fibre model if designed appropriately offers an amenable, rapid, costeffective and ethically acceptable model in which to investigate unlimited drug-target interaction/target inhibition pre-clinically.

The implantation of hollow fibres has been demonstrated in both immunocompetent (36-38) and immunodeficient mouse strains $(9-11,39,40)$ without causing immune destruction by the host or impeding the growth of tumour cells. Here we have demonstrated the comparable growth of tumour cells in hollow fibres in vivo in both immuno-competent and immunocompromised hosts. These data confirm that various mouse strains may be used for such pharmacodynamic investigations which may also provide an economic advantage.

Although we do not believe that the HFA will replace more sophisticated animal models (e.g. transgenic/orthotopic/ autochthonous/xenograft) we conclude that the hollow fibre model provides a valid method for investigating PD activity in vivo whilst successfully implementing the principles of 'reduction', 'refinement' and 'replacement', and will aid in the selection of lead molecules for further pre-clinical testing. We anticipate that the HFA will be used more readily in future years not only for reasons of amenability for PD investigation but for ethical reasons. Specifically the dramatic reduction in mouse numbers required, at a time when scientific researchers and the general public are becoming increasingly conscious of animal welfare.

\section{Acknowledgements}

We would like to acknowledge the financial support of Biotechnology \& Biological Sciences Research Council (M.S.), AstraZeneca, Alderly Edge, UK (Studentship Case Award Funding (M.S.), and Cancer Research UK (M.C.B.). Professor David Thurston, Cancer Research UK Gene Targeted Drug Design Research Group, School of Pharmacy, University of London, 29-39 Brunswick Square, London, WC1N 1AX, UK (for providing SJG-136), and Professor Jerzy Konopa, Laboratory of Molecular and Cellular Pharmacology, Department of Pharmaceutical Technology and Biochemistry, Gdansk University of Technology, 80-952 Gdansk, Narutowicza St 11/12, Poland (for providing C1311).

\section{References}

1. Russell WMS and Burch RL: The Principles of Human Experimental Technique. Methuen \& Co. Ltd., London, 1959.

2. Schechtman LM: Implementation of the 3Rs (refinement, reduction, and replacement): validation and regulatory acceptance considerations for alternative toxicological test methods. Ilar J 43 (Suppl.): S85-S94, 2002.

3. Hollingshead MG, Alley MC, Camalier RF, et al: In vivo cultivation of tumor cells in hollow fibers. Life Sci 57: 131-141, 1995.

4. Taggart BR, Harrington P and Hollingshead M: HIV hollow fiber SCID model for antiviral therapy comparison with SCID/hu model. Antiviral Res 63: 1-6, 2004.

5. Mosmann T: Rapid colorimetric assay for cellular growth and survival: application to proliferation and cytotoxicity assays. J Immunol Methods 65: 55-63, 1983. 
6. Suggitt M and Bibby M: 50 years of preclinical anti-cancer drug screening - empirical to target-driven approaches. Clin Cancer Res (Review) 11: 971-981, 2005.

7. Suggitt M, Swaine DJ, Pettit GR and Bibby MC: Characterisation of the hollow fibre assay for the determination of microtubule disruption in vivo. Clin Cancer Res 10: 6677-6685, 2004.

8. Leong CO, Suggitt M, Swaine DJ, Bibby MC, Stevens MF and Bradshaw TD: In vitro, in vivo, and in silico analyses of the antitumor activity of 2-(4-amino-3-methylphenyl)-5-fluorobenzothiazoles. Mol Cancer Ther 3: 1565-1575, 2004

9. Hall LA, Krauthauser CM, Wexler RS, Hollingshead MG, Slee AM and Kerr JS: The hollow fiber assay: continued characterization with novel approaches. Anticancer Res 20: 903-911, 2000

10. Krauthauser CM, Hall LA, Wexler RS et al: Regulation of gene expression and cell growth in vivo by tetracycline using the hollow fiber assay. Anticancer Res 21: 869-872, 2001.

11. Sadar MD, Akopian VA and Beraldi E: Characterization of a new in vivo hollow fiber model for the study of progression of prostate cancer to androgen independence. Mol Cancer Ther 1: 629-637, 2002.

12. Shnyder SD, Hasan J, Cooper PA, et al: Development of a modified hollow fibre assay for studying agents targeting the tumour neovasculature. Anticancer Res 25: 1889-1894, 2005.

13. Thurston DE: Advances in the study of pyrrolo[2,1-c][1,4]benzodiazapine (PBD) antitumour antibiotics. In: Molecular Aspects of Anticancer Drug-DNA Interactions. Macmilan. London, p53, 1993.

14. Cholody WM, Martelli S, Paradziej-Lukowicz J and Konopa J: 5-[(Aminoalkyl)amino]imidazo[4,5,1-de]acridin-6-ones as a novel class of antineoplastic agents. Synthesis and biological activity. J Med Chem 33: 49-52, 1990.

15. Giard DJ, Aaronson SA, Todaro GJ, et al: In vitro cultivation of human tumors: establishment of cell lines derived from a series of solid tumors. J Natl Cancer Inst 51: 1417-1423, 1973.

16. Collins SJ: Continuous growth and differentiation of human myeloid leukaemic cells in suspension culture. Nature 270: 347-349, 1977.

17. Fogh J: One hundred and twenty seven cultured human tumour cell lines producing tumours in mice. J Natl Cancer Inst 59: 221-226, 1977.

18. Workman P, Balmain A, Hickman JA, et al: United Kingdom Co-ordinating Committee on Cancer Research (UKCCCR) Guidelines for the Welfare of Animals in Experimental Neoplasia (2nd edition). Br J Cancer 77: 1-10, 1988.

19. Cowen SE: Vascular characterisation and evaluation of vasomanipulation to potentiate therapy in in vivo tumour models. University of Bradford, PhD Thesis, 1995.

20. Burger AM, Double JA, Konopa J and Bibby MC: Preclinical evaluation of novel imidazoacridinone derivatives with potent activity against experimental colorectal cancer. Br J Cancer 74: 1369-1374, 1996

21. Calabrese CR, Bibby MC, Double JA and Loadman PM: Pharmacokinetics and tissue distribution of the imidazoacridinone C1311 in tumour bearing mice. Cancer Chemother Pharmacol 42: 379-385, 1998.

22. Hartley JA, Spanswick VJ, Brooks N, et al: SJG-136 (NSC 694501), a novel rationally designed DNA minor groove interstrand cross-linking agent with potent and broad spectrum antitumor activity: part 1: cellular pharmacology, in vitro and initial in vivo antitumor activity. Cancer Res 64: 6693-6699, 2004.
23. Singh NP, McCoy MT, Tice RR and Schneider EL: A simple technique for quantitation of low levels of DNA damage in individual cells. Exp Cell Res 175: 184-191, 1988.

24. Szmigiero L and Studzian $\mathrm{K}: \mathrm{H}_{2} \mathrm{O}_{2}$ as a DNA fragmenting agent in the alkaline elution interstrand crosslinking and DNA-protein crosslinking assays. Anal Biochem 168: 88-93, 1988.

25. Warren AJ, Mustra DJ and Hamilton JW: Detection of mitomycin c-DNA adducts in human breast cancer cells grown in culture, as xenografted tumors in nude mice, and in biopsies of human breast cancer patient tumours as determined by $\left[{ }^{32}\right] \mathrm{P}-$ labelling. Clin Cancer Res 7: 1033-1042, 2001.

26. Alley MC, Hollingshead MG, Pacula-Cox CM, et al: SJG-136 (NSC 694501), a novel rationally designed DNA minor groove interstrand cross-linking agent with potent and broad spectrum antitumor activity: part 2: efficacy evaluations. Cancer Res 64: 6700-6706, 2004.

27. Burger AM, Jenkins TC, Double JA and Bibby MC: Cellular uptake, cytotoxicity and DNA-binding studies of the novel imidazoacridinone antineoplastic agent C1311. Br J Cancer 81: 367-375, 1999.

28. Holwell SE, Cooper PA, Grosios K, et al: Combretastatin A-1 phosphate a novel tubulin-binding agent with in vivo anti vascular effects in experimental tumours. Anticancer Res 22: 707-711, 2002

29. Scholz CC, Berger DP, Winterhalter BR, Henss $\mathrm{H}$ and Fiebig HH: Correlation of drug response in patients and in the clonogenic assay with solid human tumour xenografts. Eur J Cancer 26: 901-905, 1990.

30. Van Dyke T and Jacks T: Cancer modeling in the modern era: progress and challenges. Cell 108: 135-144, 2002.

31. Decker S, Hollingshead M, Bonomi CA, Carter JP and Sausville EA: The hollow fibre model in cancer drug screening; the NCI experience. Eur J Cancer 40: 821-826, 2004.

32. Johnson JI, Decker S, Zaharevitz D, et al: Relationships between drug activity in NCI preclinical in vitro and in vivo models and early clinical trials. Br J Cancer 84: 1424-1431, 2001

33. Parulekar WR and Eisenhauer EA: Phase I trial design for solid tumor studies of targeted, non-cytotoxic agents: theory and practice. J Natl Cancer Inst 96: 990-997, 2004.

34. Flecknell P: Replacement refinement and reduction. ALTEX 19: 7378, 2002.

35. Festing MF: Principles: the need for better experimental design. Trends Pharmacol Sci 24: 341-345, 2003.

36. Chu MY, Lipsky MH, Yee LK, et al: Predictive sensitivity of human cancer cells in vivo using semipermeable polysulfone fibers. Pharmacology 56: 318-326, 1998.

37. Jonsson E, Friberg LE, Karlsson MO, et al: Determination of drug effect on tumour cells, host animal toxicity and drug pharmacokinetics in a hollow-fibre model in rats. Cancer Chemother Pharmacol 46: 493-500, 2000

38. Jonsson E, Friberg LE, Karlsson MO, et al: In vivo activity of CHS 828 on hollow-fibre cultures of primary human tumour cells from patients. Cancer Lett 162: 193-200, 2001.

39. Mi Q, Cui B, Silva GL, et al: Pervilleines B and C, new tropane alkaloid aromatic esters that reverse the multidrug-resistance in the hollow fiber assay. Cancer Lett 184: 13-20, 2002.

40. Mi Q, Cui B, Silva GL, et al: Pervilleine A, a novel tropane alkaloid that reverses the multidrug-resistance phenotype. Cancer Res 61: 4030-4037, 2001. 